\title{
Efficacy and Safety of Tedizolid and Linezolid for the Treatment of Acute Bacterial Skin and Skin Structure Infections in Injection Drug Users: Analysis of Two Clinical Trials
}

\author{
Gregory J. Moran - Carisa De Anda - Anita F. Das · Sinikka Green • \\ Purvi Mehra $\cdot$ Philippe Prokocimer
}

Received: November 20, 2017 / Published online: September 21, 2018

(C) The Author(s) 2018

\section{ABSTRACT}

Introduction: Injection drug users (IDUs) often develop acute bacterial skin and skin structure infections (ABSSSI) and use emergency departments as their primary source for medical care. Methods: A post hoc subgroup analysis of two randomized trials examined the efficacy and safety of tedizolid in the treatment of ABSSSI in IDUs. IDUs $(n=389)$ were identified from two pooled phase 3 trials (NCT01170221, NCT01421511) in patients with ABSSSI $(n=1333)$. Patients were randomly assigned to tedizolid phosphate (200 mg once daily, 6 days)

Enhanced digital features To view enhanced digital features for this article go to https://doi.org/10.6084/ m9.figshare.7054034.

\section{G. J. Moran}

Division of Infectious Diseases, Department of Emergency Medicine, Olive View-UCLA Medical Center, Sylmar, CA, USA

C. De Anda $(\bowtie) \cdot$ P. Prokocimer

MRL, Merck \& Co., Inc., Kenilworth, NJ, USA

e-mail: carisa.de.anda@merck.com

A. F. Das

Department of Biostatistics, InClin, San Mateo, CA, USA

S. Green

eStudySite, San Diego, CA, USA

P. Mehra

Artemis Institute for Clinical Research, San Diego, CA, USA or linezolid (600 mg twice daily, 10 days). Primary endpoint was $\geq 20 \%$ reduction in lesion area from baseline at $48-72 \mathrm{~h}$. Secondary endpoints included investigator-assessed clinical and microbiological response at the post-therapy evaluation (PTE).

Results: Wound infection was more common in IDUs (52.2\%), while cellulitis/erysipelas was more common in non-IDUs (55.9\%). Most infections were due to Staphylococcus aureus (IDUs, 75.2\%; non-IDUs, 85.6\%), while oral pathogens were more prevalent in IDUs. Early clinical success rates for tedizolid and linezolid were $82.5 \%$ and $79.6 \%$ in IDUs and $81.3 \%$ and $79.3 \%$ for non-IDUs, respectively; responses at PTE were similar. Microbiological response per pathogen was similar between treatment groups. Rates of treatment-emergent adverse events (AEs) in IDUs were comparable between tedizolid (46.2\%) and linezolid (47.8\%) arms, while lower incidence of gastrointestinal AEs was observed with tedizolid (20.3\%) than with linezolid (25.1\%).

Conclusion: Efficacy and safety of tedizolid and linezolid in the treatment of ABSSSI was similar in IDUs and non-IDUs, supporting the use of oxazolidinones in treating ABSSSIs in IDUs.

Funding: Merck \& Co., Inc., Kenilworth, NJ, USA.

Keywords: ABSSSI; Injection drug user; Linezolid; Tedizolid 


\section{INTRODUCTION}

Acute bacterial skin and skin structure infections (ABSSSI) are among the most common reasons for visits to the emergency department [1]. One group at particularly high risk for ABSSSI is injection drug users (IDUs) [2-5]. The high incidence and the high rate of recurrence of ABSSSI in this population are likely due to frequent, repeated injections, injection method (subcutaneous injection), and/or drug mixture (cocaine and heroin, often with contaminants). The lack of aseptic injection methods, including the practice of needle licking, contaminates the injection site and increases the risk for skin infections $[2,3,5,6]$. Subcutaneous injection has been strongly associated with the development of purulent infections and cellulitis due to the localized introduction of contaminants, irritants, and bacteria directly into the tissue $[7,8]$. Injection of cocaine and heroin may also increase the risk for ABSSSI because it induces localized irritation and tissue reaction that promote the development of infections [8].

Among IDUs, the most commonly identified causative pathogens for ABSSSI are Staphylococcus aureus-including methicillin-resistant $S$. aureus (MRSA) [3, 4, 9, 10]-and streptococcal species [3]. However, the bacteriology of ABSSSI in IDUs can be diverse and polymicrobic with infections from nontraditional pathogenssuch as those present in the oral flora-commonly seen $[4,10]$.

Injection drug users often use emergency departments as their primary source for medical care, with ABSSSI a leading reason for presentation $[2,4]$. Physicians may be faced with many challenges when treating IDUs including poor compliance, delays in seeking medical care, and recurring infections $[2,3,7]$. Additionally, many IDUs have comorbid mental health illnesses and hepatitis C or HIV coinfection, which may complicate treatment options (e.g., limited mental capacity, immunosuppression, adherence to therapy) [2, 11-13]. Given the numerous challenges associated with treating ABSSSI in IDUs, better understanding of patient and disease characteristics and clinical outcomes in IDUs compared with non-IDUs may help guide the best treatment option for this patient population.

Tedizolid, the active moiety of tedizolid phosphate, is a novel oxazolidinone antibacterial with potent activity against a wide range of Gram-positive pathogens, including MRSA and vancomycin-resistant enterococci [14-16]. Two phase 3 trials, ESTABLISH-1 and ESTABLISH-2, demonstrated the noninferior efficacy of tedizolid (200 mg daily for 6 days) compared with linezolid (600 mg twice daily for 10 days) in patients with ABSSSI [17, 18]. This post hoc subgroup analysis, using pooled data from the two phase 3 ABSSSI clinical trials, aims to assess the safety and efficacy of tedizolid and linezolid in IDUs and non-IDUs.

\section{METHODS}

\section{Study Design and Setting}

All procedures followed were in accordance with the ethical standards of the responsible committee on human experimentation (institutional and national) and with the Helsinki Declaration of 1964, as revised in 2013. Informed consent was obtained from all patients for being included in the study. The study was conducted in accordance with the principles of Good Clinical Practice and was approved by the appropriate institutional review boards and regulatory agencies. ESTABLISH-1 and ESTABLISH-2 were randomized, double-blind, double-dummy, multicenter, multinational, noninferiority, phase 3 clinical trials in patients with ABSSSI. Patients had to have suspected or documented Gram-positive infection from baseline Gram stain or culture to be included in the study. Patients with cellulitis/erysipelas or abscess were not enrolled if there was suspected Gram-negative involvement. Concomitant Gram-negative coverage was only allowed with infected wounds. Methods and results of the individual studies and pooled analysis have been previously published [17-19]. These trials are registered at ClinicalTrials.gov NCT01421511). 


\section{Selection of Participants}

The trials enrolled patients older than 18 years (ESTABLISH-1) or older than 12 years (ESTABLISH-2) with ABSSSI (cellulitis/erysipelas, wound infection or major cutaneous abscess). Full details of patient eligibility criteria have been published $[17,18]$. The pooled intent-totreat (ITT) population from both clinical trials was classified into IDU and non-IDU subgroups based on injection drug use, and all endpoints were evaluated between these groups. Patients who disclosed that they were current or recent users of injection drugs at screening were included in the IDU subpopulation.

\section{Interventions}

Patients received $200 \mathrm{mg}$ tedizolid phosphate once daily for 6 days or $600 \mathrm{mg}$ linezolid twice daily for 10 days. Patients in ESTABLISH-1 received oral drug. Patients in ESTABLISH-2 received intravenous treatment for 1 day and could subsequently be switched to oral drug at the investigator's discretion if they met two or more of the following criteria: no increase from baseline in primary lesion area, temperature lower than $37.7^{\circ} \mathrm{C}$, no worsening of local signs and symptoms, or improvement of local signs and symptoms at the primary infection site.

\section{Methods and Measurements}

Baseline assessments were performed within $24 \mathrm{~h}$ before the first dose of study drug (day 1 ). Patients were evaluated on day 1 , day $2,48-72 \mathrm{~h}$ after the first dose, day 7 , and day 11 [end-oftreatment (EOT) visit]; at the post-therapy evaluation (PTE) visit (7-14 days after the EOT visit); and at the late follow-up (LFU) visit (18-25 days after the EOT visit). Intravenous treatment was delivered in a health care setting, but patients were permitted to take oral study drug at home.

Bacterial pathogens were identified from a blood culture or from culture of a microbiological sample obtained from the primary lesion site by needle aspiration, biopsy, deep swab, incision or other surgical techniques. Pathogens (genus and species) were identified by the central laboratory; local laboratory data were used if central laboratory data were not available.

\section{Outcome Measures}

The primary endpoint was $a \geq 20 \%$ reduction in lesion area [length $\times$ width of erythema, edema or induration (in ESTABLISH-1; only erythema was used to determine lesion size)] without receipt of rescue antibiotics. Early clinical response was assessed $48-72 \mathrm{~h}$ after the first dose of study drug; patients who had received nonstudy systemic antibacterial agents for any reason within $72 \mathrm{~h}$ of the first dose were considered nonresponders, per US Food and Drug Administration guidelines [20]. A key secondary endpoint was late response, which involved investigator-assessed clinical response at the PTE [7-14 days after the end of therapy (EOT; 11 days after the start of study drug)], per European Medicines Agency guidelines [21].

Microbiological response per pathogen at EOT and PTE was also analyzed. A favorable microbiological response was defined as eradication (absence of original baseline pathogens) and presumed eradication (no source specimen to culture in a patient assessed by the investigator as a clinical success).

Safety evaluations included adverse events (AEs), clinical laboratory assessments, vital signs, electrocardiograms, and physical examination.

\section{Analyses}

The ITT population included all randomly assigned patients. Patients with missing data and patients who did not receive study drug were defined as having an indeterminate response and were considered nonresponders for the primary efficacy endpoint. The microbiological ITT (MITT) population included patients with $\geq 1$ Gram-positive ABSSSI pathogen isolated at baseline. The clinically evaluable (CE) population included all patients in the ITT analysis set who complied with the protocol and had no major violations. The CE population at PTE (CE-PTE) included patients who 


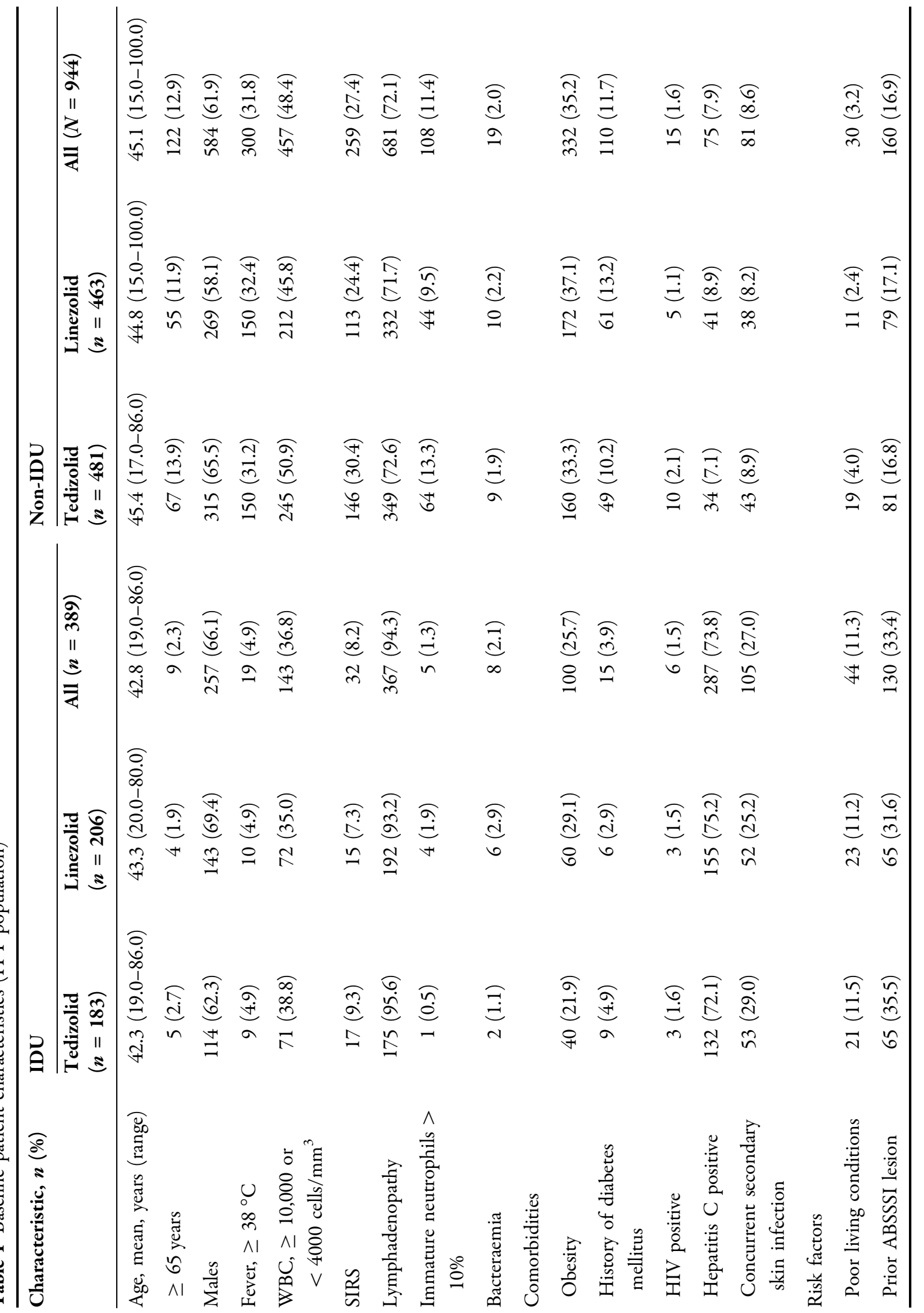




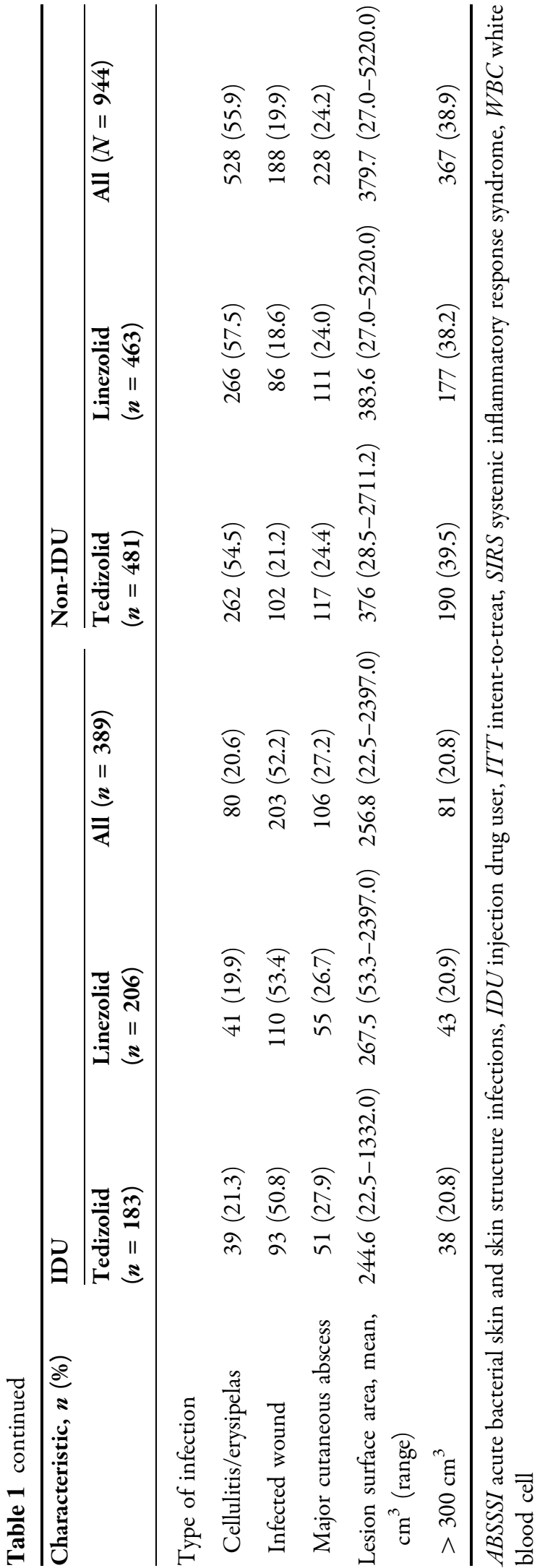

completed the study through the PTE assessment without any concomitant systemic antibiotic therapy or other confounding events or factors. The microbiologically evaluable at PTE (ME-PTE) population included all patients in the MITT analysis set who were also in the CE-PTE analysis set. The microbiologically evaluable at end-of-therapy (ME-EOT) population included all patients in the MITT and clinically evaluable at end of therapy (CE-EOT) populations. The safety population included all patients from the MITT population who received $\geq 1$ dose of the study drug.

Summaries included frequency and percentages for categorical data, frequency and median for ordinal data, and frequency, mean, standard deviation, and median, minimum and maximum, for quantitative data. Version 9.2 (or higher) of SAS statistical software package was used to provide all summaries and statistical analyses. For both primary and secondary outcomes, 95\% confidence intervals (CIs) were adjusted for the study and were calculated using the methodology of Miettinen and Nurminen [22] to determine the differences in response rates between tedizolid and linezolid treatment arms.

\section{RESULTS}

\section{Characteristics of Study Subjects}

A total of 1333 patients (664 treated with tedizolid and 669 treated with linezolid) made up the ITT population. Approximately $30 \%$ of the pooled ITT population were IDUs: $27.6 \%$ (183/ 664) were in the tedizolid group and $30.8 \%$ $(206 / 669)$ were in the linezolid group. Fewer IDUs were elderly (aged $\geq 65$ years) (Table 1 ). Overall, IDUs had a lower incidence of traditional systemic signs and symptoms of infection than non-IDUs, including fever [4.9\% (19/389) vs. $31.8 \%(300 / 944)]$, abnormal white blood cell count [36.8\% (143/389) vs. $48.4 \%$ (457/944)], systemic inflammatory response syndrome [SIRS; $8.2 \%(32 / 389)$ vs. $27.4 \%(259 / 944)]$, and immature neutrophils [1.3\% (5/389) vs. $11.4 \%$ (108/944)], respectively. Lymphadenopathy, however, was more prevalent in IDUs [94.3\% 
Table 2 Baseline pathogens by injection drug use (MITT population)

\begin{tabular}{|c|c|c|c|c|}
\hline \multirow[t]{2}{*}{ Pathogens, $n(\%)$} & \multicolumn{2}{|c|}{ IDU $(N=282)$} & \multicolumn{2}{|c|}{ Non-IDU $(N=536)$} \\
\hline & $\begin{array}{l}\text { Tedizolid } \\
(n=129)\end{array}$ & $\begin{array}{l}\text { Linezolid } \\
(n=153)\end{array}$ & $\begin{array}{l}\text { Tedizolid } \\
(n=277)\end{array}$ & $\begin{array}{l}\text { Linezolid } \\
(n=259)\end{array}$ \\
\hline Gram-positive aerobes & $125(96.9)$ & $150(98.0)$ & $274(98.9)$ & $255(98.5)$ \\
\hline Staphylococcus aureus & $95(73.6)$ & $117(76.5)$ & $234(84.5)$ & $225(86.9)$ \\
\hline $\mathrm{MRSA}^{\mathrm{a}}$ & $38(40.0)$ & $46(39.3)$ & $103(44.0)$ & $100(44.4)$ \\
\hline MSSA $^{\mathrm{a}}$ & $57(60.0)$ & $72(61.5)$ & $131(56.0)$ & $126(56.0)$ \\
\hline Streptococcus pyogenes & $0(0.0)$ & $1(0.7)$ & $33(11.9)$ & $19(7.3)$ \\
\hline Streptococcus anginosus group & $26(20.2)$ & $22(14.4)$ & $4(1.4)$ & $6(2.3)$ \\
\hline Streptococcus mitis & $2(1.6)$ & $7(4.6)$ & - & - \\
\hline Streptococcus salivarius & $2(1.6)$ & $2(1.3)$ & - & - \\
\hline Streptococcus sanguinis & $4(3.1)$ & $2(1.3)$ & - & - \\
\hline Streptococcus viridans group & $3(2.3)$ & $7(4.6)$ & - & - \\
\hline Gram-positive anaerobes & $7(5.4)$ & $6(3.9)$ & $3(1.1)$ & $7(2.7)$ \\
\hline Clostridium perfringens & $4(3.1)$ & $2(1.3)$ & - & - \\
\hline Gram-negative aerobes & $4(3.1)$ & $5(3.3)$ & $5(1.8)$ & $2(0.8)$ \\
\hline $\begin{array}{l}\text { Monomicrobial baseline } \\
\text { infection }\end{array}$ & $105(81.4)$ & $131(85.6)$ & $250(90.3)$ & $231(89.2)$ \\
\hline Polymicrobial baseline infection & $24(18.6)$ & $22(14.4)$ & $27(9.7)$ & $28(10.8)$ \\
\hline
\end{tabular}

$I D U$ injection drug user, MITT microbiological intent-to-treat, MRSA methicillin-resistant $S$. aureus, MSSA methicillinsusceptible $S$. aureus, non-IDU non-injection drug user

${ }^{a}$ Percentage is calculated with total number of $S$. aureus in each group as the denominator

(367/389)] than in non-IDUs [72.1\% (681/944)]. Risk factors, including poor living conditions [11.3\% (44/389) vs. 3.2\% (30/944)] and previous ABSSSI lesion [33.4\% (130/389) vs. 16.9\% (160/ 944); Table 1], respectively, were more common in IDUs than in non-IDUs. Hepatitis $\mathrm{C}$ and concurrent secondary skin infection were approximately nine times [73.8\% (287/389) vs. $7.9 \%(75 / 944)]$ and three times [27.0\% (105/ $389)$ vs. $8.6 \%(81 / 944)]$ more prevalent in IDUs than in non-IDUs, respectively (Table 1 ). However, the incidence of each of these comorbidities was similar between the tedizolid and linezolid treatment groups for both IDUs and non-IDUs (Table 1).

The distribution of ABSSSI types differed between IDUs and non-IDUs in both treatment groups. Wound infections, often injection-site infections, were the most common ABSSSI in IDUs [tedizolid, 50.8\% (93/183); linezolid, $53.4 \%(110 / 206)]$, whereas, in non-IDUs, cellulitis/erysipelas was more prevalent [tedizolid, 54.5\% (262/481); linezolid, 57.5\% (266/463); Table 1].

Patients who used injection drugs presented with a more diverse spectrum of baseline pathogens, including organisms not traditionally associated with ABSSSI, and more frequently had polymicrobial Gram-positive infections (Table 2). Among IDUs and nonIDUs, the most common baseline pathogen was S. aureus $[75.2 \%(212 / 282)$ and $85.6 \%(459 /$ $536)$, respectively]; the prevalence of methicillin-resistant and methicillin-susceptible $S$. aureus was similar between IDUs [39.6\% (84/ $212)$ and $60.8 \%(129 / 212)$, respectively] and 


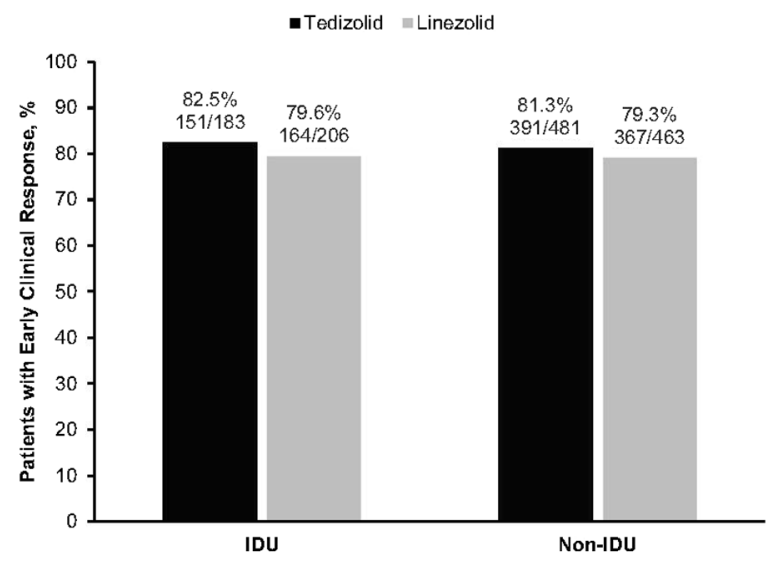

Fig. 1 Early clinical response at the 48- to 72 -h visits by injection drug use in the intent-to-treat population. Early clinical response was defined as $\geq 20 \%$ reduction in lesion size. Early clinical response rates for the tedizolid and linezolid treatment groups were similar in IDUs and nonIDUs. IDUs injection drug users, non-IDUs non-injection drug users

non-IDUs [44.2\% (203/459) and 56.0\% (257/ 459), respectively]. However, Streptococcus anginosus Group [17\% (48/282) in IDUs and 1.9\% $(10 / 536)$ in non-IDUs] and streptococcal species that generally form part of the oral flora [including $S$. mitis, $S$. salivarius, $S$. sanguinis, and $S$. viridans Group; $10.3 \%(29 / 282)$ vs. $0.0 \%$ in nonIDUs] were more frequently observed in patients who used injection drugs (Table 2). Infections due to $S$. pyogenes were more frequent in non-IDUs $[9.7 \%(52 / 536)]$ than IDUs $[0.4 \%$ $(1 / 282)]$.

\section{Efficacy}

Early clinical response rates in the ITT population at the 48- to 72 -h visits were similar in IDUs treated with tedizolid $[82.5 \%(151 / 183)]$ or linezolid [79.6\% (164/206); Fig. 1]. For IDUs, the treatment difference between tedizolid and linezolid was $2.9 \%$ (95\% CI - 4.9, 10.7). Similarly, for non-IDUs, early clinical response was comparable between tedizolid-treated patients [81.3\% (391/481)] and linezolid-treated patients [79.3\% (367/463)] (Fig. 1). Early response rates
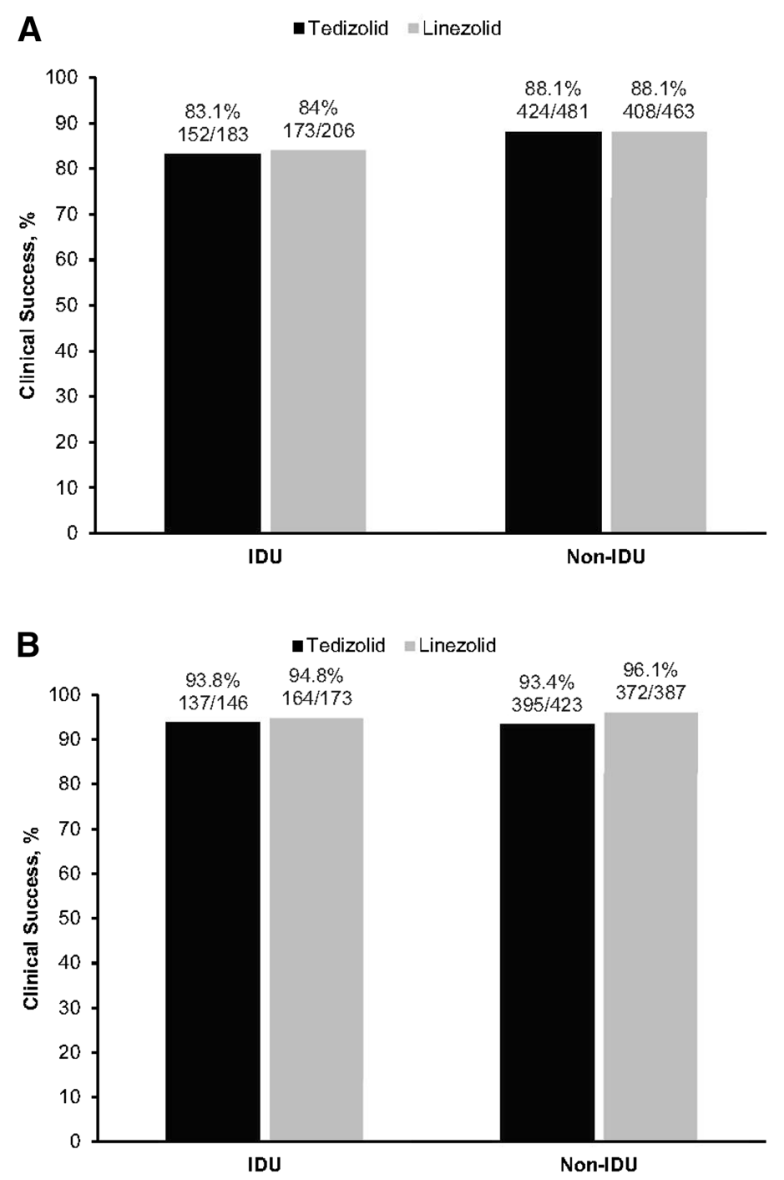

Fig. 2 Clinical success rates at the PTE by injection drug use in the a ITT population and the $\mathbf{b}$ CE-PTE population. No differences in clinical response rates at the PTE were observed between tedizolid and linezolid treatment or between IDUs and non-IDUs in the ITT or CE-PTE population. $C E$ clinically evaluable, $I D U s$ injection drug users, ITT intent-to-treat, non-IDUs noninjection drug users, PTE post-therapy evaluation

did not differ between IDUs and non-IDUs (Fig. 1).

In the ITT population, the rates of clinical success based on investigator assessment of clinical response at the PTE visit were similar between IDUs and non-IDUs treated with tedizolid [83.1\% (152/183) and 88.1\% (424/481), respectively] or linezolid [84.0\% $(173 / 206)$ and $88.1 \%$ (408/463), respectively] (Fig. 2a). The treatment difference between tedizolid and 
Table 3 Rates of favorable microbiological response per baseline pathogen at EOT visit

\begin{tabular}{|c|c|c|c|c|c|c|c|c|}
\hline \multirow{3}{*}{$\begin{array}{l}\text { Favorable response, } \\
n / N(\%)\end{array}$} & \multicolumn{4}{|c|}{ MITT population } & \multicolumn{4}{|c|}{ ME-EOT population } \\
\hline & \multicolumn{2}{|l|}{$\overline{\text { IDU }}$} & \multicolumn{2}{|l|}{ Non-IDU } & \multicolumn{2}{|l|}{$\overline{\text { IDU }}$} & \multicolumn{2}{|l|}{ Non-IDU } \\
\hline & Tedizolid & Linezolid & Tedizolid & $\overline{\text { Linezolid }}$ & Tedizolid & $\overline{\text { Linezolid }}$ & Tedizolid & Linezolid \\
\hline $\begin{array}{l}\text { Gram-positive } \\
\text { aerobes }\end{array}$ & $\begin{array}{r}129 / 151 \\
(85.4)\end{array}$ & $\begin{array}{c}145 / 170 \\
(85.3)\end{array}$ & $\begin{array}{c}301 / 332 \\
(90.7)\end{array}$ & $\begin{array}{r}276 / 301 \\
(91.7)\end{array}$ & $\begin{array}{r}116 / 121 \\
(95.9)\end{array}$ & $\begin{array}{c}136 / 142 \\
(95.8)\end{array}$ & $\begin{array}{c}270 / 282 \\
(95.7)\end{array}$ & $\begin{array}{c}245 / 246 \\
(99.6)\end{array}$ \\
\hline $\begin{array}{l}\text { Staphylococcus } \\
\text { aureus }\end{array}$ & $\begin{array}{r}92 / 100 \\
(92.0)\end{array}$ & $\begin{array}{r}105 / 120 \\
(87.5)\end{array}$ & $\begin{array}{r}237 / 261 \\
(90.8)\end{array}$ & $\begin{array}{r}226 / 246 \\
(91.9)\end{array}$ & $\begin{array}{l}83 / 87 \\
(95.4)\end{array}$ & $\begin{array}{r}97 / 100 \\
\quad(97.0)\end{array}$ & $\begin{array}{c}215 / 224 \\
(96.0)\end{array}$ & $\begin{array}{r}200 / 201 \\
(99.5)\end{array}$ \\
\hline MRSA & $\begin{array}{l}35 / 41 \\
\quad(85.4)\end{array}$ & $\begin{array}{l}38 / 47 \\
(80.9)\end{array}$ & $\begin{array}{r}96 / 111 \\
(86.5)\end{array}$ & $\begin{array}{r}96 / 110 \\
(87.3)\end{array}$ & $\begin{array}{l}33 / 36 \\
(91.7)\end{array}$ & $\begin{array}{l}34 / 36 \\
\quad(94.4)\end{array}$ & $\begin{array}{l}90 / 96 \\
(93.8)\end{array}$ & $\begin{array}{l}86 / 87 \\
(98.9)\end{array}$ \\
\hline MSSA & $\begin{array}{l}57 / 59 \\
\quad(96.6)\end{array}$ & $\begin{array}{l}67 / 73 \\
\quad(91.8)\end{array}$ & $\begin{array}{r}141 / 150 \\
(94.0)\end{array}$ & $\begin{array}{r}129 / 135 \\
(95.6)\end{array}$ & $\begin{array}{l}50 / 51 \\
\quad(98.0)\end{array}$ & $\begin{array}{l}63 / 64 \\
\quad(98.4)\end{array}$ & $\begin{array}{r}125 / 128 \\
(97.7)\end{array}$ & $\begin{array}{r}113 / 113 \\
(100.0)\end{array}$ \\
\hline $\begin{array}{l}\text { Streptococcus } \\
\text { pyogenes }\end{array}$ & - & $\begin{array}{l}1 / 1 \\
\quad(100.0)\end{array}$ & $\begin{array}{l}38 / 39 \\
(97.4)\end{array}$ & $\begin{array}{l}19 / 20 \\
\quad(95.0)\end{array}$ & - & $\begin{array}{l}1 / 1 \\
\quad(100.0)\end{array}$ & $\begin{array}{l}32 / 32 \\
\quad(100.0)\end{array}$ & $\begin{array}{l}18 / 18 \\
(100.0)\end{array}$ \\
\hline $\begin{array}{l}\text { Streptococcus } \\
\text { anginosus group }\end{array}$ & $\begin{array}{l}18 / 26 \\
(69.2)\end{array}$ & $\begin{array}{l}20 / 22 \\
\quad(90.9)\end{array}$ & $\begin{array}{l}4 / 4 \\
\quad(100.0)\end{array}$ & $5 / 6(83.3)$ & $\begin{array}{l}16 / 17 \\
(94.1)\end{array}$ & $\begin{array}{l}20 / 21 \\
\quad(95.2)\end{array}$ & $\begin{array}{l}3 / 3 \\
\quad(100.0)\end{array}$ & $\begin{array}{l}3 / 3 \\
\quad(100.0)\end{array}$ \\
\hline Streptococcus mitis & $\begin{array}{l}2 / 2 \\
\quad(100.0)\end{array}$ & $6 / 7(85.7)$ & - & - & $\begin{array}{l}2 / 2 \\
\quad(100.0)\end{array}$ & $\begin{array}{l}5 / 5 \\
\quad(100.0)\end{array}$ & - & - \\
\hline $\begin{array}{l}\text { Streptococcus } \\
\text { salivarius }\end{array}$ & $1 / 2(50.0)$ & $\begin{array}{l}2 / 2 \\
\quad(100.0)\end{array}$ & - & - & $\begin{array}{l}1 / 1 \\
\quad(100.0)\end{array}$ & $\begin{array}{l}2 / 2 \\
\quad(100.0)\end{array}$ & - & - \\
\hline $\begin{array}{l}\text { Streptococcus } \\
\text { sanguinis }\end{array}$ & $2 / 4(50.0)$ & $0 / 2(0.0)$ & - & - & - & - & - & - \\
\hline $\begin{array}{l}\text { Streptococcus } \\
\text { viridans group }\end{array}$ & $2 / 3(66.7)$ & $3 / 8(37.5)$ & - & - & $\begin{array}{l}2 / 2 \\
\quad(100.0)\end{array}$ & $3 / 5(60.0)$ & - & - \\
\hline $\begin{array}{c}\text { Gram-positive } \\
\text { anaerobes }\end{array}$ & $7 / 8(87.5)$ & $4 / 7(57.1)$ & $\begin{array}{l}4 / 4 \\
\quad(100.0)\end{array}$ & $8 / 9(88.9)$ & $\begin{array}{l}5 / 5 \\
\quad(100.0)\end{array}$ & $4 / 6(66.7)$ & $\begin{array}{l}3 / 3 \\
\quad(100.0)\end{array}$ & $\begin{array}{l}5 / 5 \\
\quad(100.0)\end{array}$ \\
\hline $\begin{array}{l}\text { Clostridium } \\
\text { perfringens }\end{array}$ & $\begin{array}{l}4 / 4 \\
\qquad(100.0)\end{array}$ & $1 / 2(50.0)$ & - & - & $\begin{array}{l}4 / 4 \\
\quad(100.0)\end{array}$ & $\begin{array}{l}1 / 1 \\
\quad(100.0)\end{array}$ & - & - \\
\hline $\begin{array}{l}\text { Gram-negative } \\
\text { aerobes }\end{array}$ & $4 / 6(66.7)$ & $5 / 6(83.3)$ & $3 / 8(37.5)$ & $\begin{array}{l}2 / 2 \\
\quad(100.0)\end{array}$ & $\begin{array}{l}4 / 4 \\
\quad(100.0)\end{array}$ & $\begin{array}{l}5 / 5 \\
\quad(100.0)\end{array}$ & $2 / 4(50.0)$ & $\begin{array}{l}1 / 1 \\
\quad(100.0)\end{array}$ \\
\hline
\end{tabular}

$E O T$ end of treatment, IDU injection drug user, MITT microbiological intent-to-treat, $M E$ microbiologically evaluable, $M R S A$ methicillin-resistant $S$. aureus, MSSA methicillin-susceptible $S$. aureus, non-IDU non-injection drug user

linezolid was $-0.9 \%(95 \%$ CI $-8.3,6.5)$ for IDUs. Clinical success rates in the CE-PTE population were also comparable between IDUs treated with tedizolid and linezolid (Fig. 2b), with a treatment difference of $-1.0 \%$ (95\% CI $-6.8,4.2)$ in IDUs. Similar clinical success rates were observed for IDUs and non-IDUs at the
PTE visit in both the ITT (83.1-88.1\%) and the CE-PTE (93.4-96.1\%) populations (Fig. 2a, b).

Rates of favorable microbiological response per baseline pathogen at the EOT visit for the MITT and ME-EOT populations are presented in Table 3. Rates were generally similar between both treatment groups in IDUs and non-IDUs 

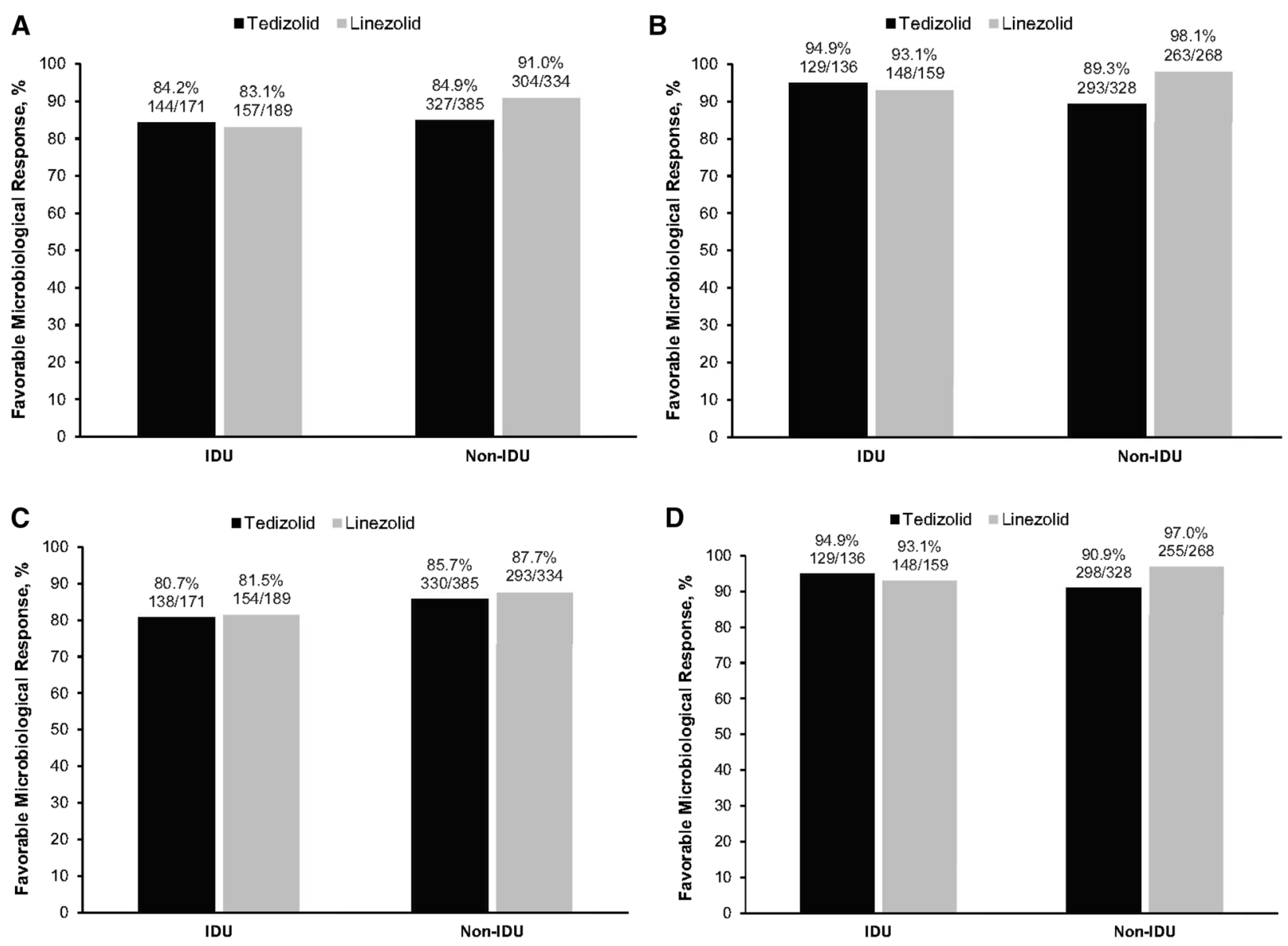

Fig. 3 Favorable microbiological response by injection drug use. a EOT evaluation for the MITT population. b EOT evaluation for the ME-EOT population. c PTE for the MITT population. d PTE evaluation for the ME-PTE population. No difference in favorable microbiological response was observed between tedizolid and linezolid

from the MITT and ME-EOT populations at EOT (Fig. 3a, b). Microbiological response to $S$. anginosus was lower for tedizolid than for linezolid in IDUs from the MITT population at EOT. However, at the PTE visit, high response rates were observed with both treatments in IDUs and non-IDUs ( $>80 \%$ positive response in the MITT population and $>90 \%$ positive response in the ME-PTE population) (Fig. 3c, d).

\section{Safety}

The incidence of treatment-emergent adverse events (TEAEs) was slightly elevated in IDUs

treatment in IDUs and non-IDUs in these populations. $E O T$ end-of-therapy, IDUs injection drug users, $M E$ microbiologically evaluable, MITT microbiological intentto-treat, non-IDUs non-injection drug users, PTE posttherapy evaluation

compared with non-IDUs. TEAEs occurred in $46.2 \%(84 / 182)$ of tedizolid patients and $47.8 \%$ $(97 / 203)$ of linezolid patients who used injection drugs; in non-IDUs, $41.5 \%(199 / 480)$ of tedizolid patients and $41.2 \%(189 / 459)$ of linezolid patients reported experiencing any TEAE (Table 4). TEAEs under the grouping "infections and infestations" were more common among IDUs than non-IDUs, particularly new, secondary abscess or cellulitis. In the tedizolid group, $8.2 \%(15 / 182)$ of IDUs and $4.2 \%$ (20/ $480)$ of non-IDUs experienced secondary abscess; $4.4 \% \quad(8 / 182)$ and $1.9 \%$ (9/480), respectively, had secondary cellulitis. Results were similar in the linezolid group: 6.9\% (14/ 
Table 4 Summary of adverse events by injection drug use (safety population)

\begin{tabular}{|c|c|c|c|c|}
\hline \multirow[t]{2}{*}{ Adverse event, $n$ (\%) } & \multicolumn{2}{|c|}{ IDU $(N=385)$} & \multicolumn{2}{|c|}{ Non-IDU $(N=939)$} \\
\hline & $\begin{array}{l}\text { Tedizolid } \\
(n=182)\end{array}$ & $\begin{array}{l}\text { Linezolid } \\
(n=203)\end{array}$ & $\begin{array}{l}\text { Tedizolid } \\
(n=480)\end{array}$ & $\begin{array}{l}\text { Linezolid } \\
(n=459)\end{array}$ \\
\hline All AEs & $86(47.3)$ & $97(47.8)$ & $203(42.3)$ & $191(41.6)$ \\
\hline Any TEAE & $84(46.2)$ & $97(47.8)$ & $199(41.5)$ & $189(41.2)$ \\
\hline Drug-related TEAE & $50(27.5)$ & $68(33.5)$ & $98(20.4)$ & $117(25.5)$ \\
\hline Moderate/severe TEAE & $28(15.4)$ & $42(20.7)$ & $60(12.5)$ & $51(11.1)$ \\
\hline Serious TEAE & $5(2.7)$ & $4(2.0)$ & $7(1.5)$ & $9(2.0)$ \\
\hline Drug-related serious TEAE & $0(0.0)$ & $1(0.5)$ & $0(0.0)$ & $1(0.2)$ \\
\hline $\begin{array}{l}\text { TEAE leading to } \\
\text { discontinuation }\end{array}$ & $2(1.1)$ & $3(1.5)$ & $1(0.2)$ & $3(0.7)$ \\
\hline TEAE leading to death & $1(0.5)$ & $0(0.0)$ & $1(0.2)$ & $1(0.2)$ \\
\hline \multicolumn{5}{|l|}{ TEAE ( $\geq 2 \%$ in frequency) } \\
\hline Abscess & $15(8.2)$ & $14(6.9)$ & $20(4.2)$ & $12(2.6)$ \\
\hline Cellulitis & $8(4.4)$ & $7(3.4)$ & $9(1.9)$ & $7(1.5)$ \\
\hline Diarrhoea & $9(4.9)$ & $12(5.9)$ & $17(3.5)$ & $23(5.0)$ \\
\hline Dizziness & $5(2.7)$ & $4(2.0)$ & $7(1.5)$ & $10(2.2)$ \\
\hline Fatigue & $0(0.0)$ & $2(1.0)$ & $9(1.9)$ & $10(2.2)$ \\
\hline Headache & $6(3.3)$ & $10(4.9)$ & $35(7.3)$ & $29(6.3)$ \\
\hline Nausea & $17(9.3)$ & $33(16.3)$ & $37(7.7)$ & $48(10.5)$ \\
\hline Pruritus & $1(0.5)$ & $5(2.5)$ & $2(0.4)$ & $4(0.9)$ \\
\hline Vomiting & $11(6.0)$ & $13(6.4)$ & $8(1.7)$ & $24(5.2)$ \\
\hline Wound infection & $5(2.7)$ & $2(1.0)$ & $1(0.2)$ & $0(0.0)$ \\
\hline
\end{tabular}

Patients reporting an AE were counted only once

$A E$ adverse event, IDU injection drug user, non-IDU non-injection drug user, TEAE treatment-emergent adverse event

203) and 2.6\% (12/459) of IDUs and non-IDUs, respectively, had secondary abscess and $3.4 \%$ (7/203) and $1.5 \%$ (7/459), respectively, had secondary cellulitis.

Among the most frequently reported TEAEs ( $\geq 2 \%$, gastrointestinal (GI) disorders (including nausea, vomiting, and diarrhea) were most common and were higher in the linezolid arm in both IDUs and non-IDUs. GI TEAEs in IDUs were reported in $20.3 \%(37 / 182)$ of the tedizolid group compared with $25.1 \%(51 / 203)$ of the linezolid group, which was slightly higher than rates reported in non-IDUs [tedizolid, 14.4\%
(69/480); linezolid, 22.0\% (101/459)]. Three deaths occurred during the study; one of those who died was a patient actively using injection drugs. This patient developed septic shock with multiorgan failure and died 46 days after receiving the final dose of tedizolid. All deaths were considered unrelated to the study drug [19].

\section{DISCUSSION}

ABSSSI is a leading reason that IDUs seek medical treatment $[2,4]$. Treatment of this patient 
group frequently rests with emergency physicians as many IDUs utilize emergency departments as their primary source of medical care $[2,4]$. Effective antibacterial treatment relies heavily on the appropriateness of initial therapy and first-line treatment in the emergency department is critical to ensuring successful outcomes in this patient population.

In this post hoc subgroup analysis from two phase 3 clinical trials of tedizolid, IDUs overall had fewer traditional systemic signs and symptoms of infection (fever, abnormal white blood cell counts, SIRS, and immature neutrophils) than non-IDUs. However, lymphadenopathy, hepatitis $\mathrm{C}$, poor living conditions, and past ABSSSI lesions were more prevalent in IDUs, consistent with reported conditions known to be common in this population [7, 13, 23, 24]. The higher incidence of secondary skin infections and hepatitis $C$ is unsurprising given that IDUs repeatedly inject into various anatomic sites which all have the potential for infection.

The distribution of ABSSSI types differed between IDUs and non-IDUs. As would be expected from repeated injections, more than half of IDUs had wound infections, whereas non-IDUs predominantly had cellulitis/erysipelas, similar to what was observed in the overall ITT population [19]. It is possible that some infections in IDUs classified as cellulitis/erysipelas or abscess were related to an injection site and might therefore be considered wound infections or vice versa.

The majority of ABSSSI in both IDUs and non-IDUs were due to $S$. aureus. However, infections in IDUs were associated with a more diverse range of bacterial species not typically causative of ABSSSI, specifically those of the oral flora. These species increase the risk for infection from oral streptococcal and Gram-positive anaerobic species $[6,7]$. The findings from this study are consistent with previous reports that found $S$. aureus to be the predominant causative pathogen in ABSSSI with oral pathogens constituting higher proportions among IDUs $[4,10]$.

Despite differences in baseline patient and clinical characteristics, treatment outcomes in both IDUs and non-IDUs were comparable with tedizolid and linezolid at both the early evaluation and the PTE. Early clinical response approximated $80 \%$ for IDUs and non-IDUs in both treatment arms. This rate was similar to that observed in the overall, pooled ITT population, as reported [19]. Investigator-assessed clinical response at PTE was also comparable between treatment arms and IDU/non-IDU patient populations. Similarities in clinical outcomes suggest that complicating factors associated with injection drug use did not greatly impact the efficacy profile of tedizolid or linezolid in treating ABSSSI. The microbiological response of IDUs was also generally similar to that of non-IDUs in the MITT and ME populations, despite the greater diversity of pathogens in the IDU group and regardless of treatment arm. At EOT and PTE, high response rates were observed for both treatments in IDUs, and $>90 \%$ of pathogens were eradicated or presumed eradicated with either treatment in both IDUs and non-IDUs in the ME population.

Overall, tedizolid and linezolid were well tolerated in IDUs, although the rate of TEAEs was slightly higher in IDUs than in non-IDUs. In both treatment groups, IDUs experienced secondary abscesses and secondary cellulitis more often than non-IDUs, although this could be associated with new infections caused by repeated needle use in other locations. GI disorders also occurred at a higher frequency in IDUs, with a numerically greater incidence in the linezolid group than in the tedizolid group, consistent with safety results observed in the overall ITT population [19]. These GI TEAEs generally occurred within the first 6 days of treatment. As such, they were likely the result of pharmacologic differences between linezolid and tedizolid and not a result of the shorter course of therapy with tedizolid phosphate [19].

The high-risk lifestyle of IDUs, often coupled with increased rates of reinfection and concomitant mental health issues, makes compliance a major concern in this treatment population [2, 11, 13, 25]. In a systematic review of patient adherence to oral antibacterials, medical compliance was higher in patients prescribed once-daily, short-course oral therapy than in patients prescribed longer and more frequent regimens [26]. The convenience and effectiveness of a once-daily, short-course oral 
treatment such as tedizolid phosphate may be particularly beneficial in this population. Reducing pill burden has the potential to increase treatment compliance and optimize outcomes in a real-world setting. IDUs may also be at risk for serotonin syndrome, a potentially life-threatening reaction caused by overactivation of the serotonergic system [27]. Many cases of serotonin syndrome are caused by unintended drug-drug interactions, and it is known that some drugs of abuse can lead to serotonin toxicity [27]. Tedizolid may be less likely to cause serotonin syndrome than linezolid, though further research is required to support this hypothesis [28, 29].

Several limitations should be considered when evaluating the results of this study. This was a post hoc subgroup analysis of a patient subpopulation and might have lacked sufficient power to detect differences between IDUs and non-IDUs. The study relied on self-reporting of injection drug use, which might have been subject to underreporting. In addition, the two treatment groups within the IDU and non-IDU subgroups might not have been balanced with respect to all prognostic factors.

\section{CONCLUSION}

In summary, although IDUs present with treatment challenges not typically seen in nonIDUs, clinical outcomes were similar for both patient populations. Both tedizolid and linezolid were well tolerated; however, fewer GI AEs were associated with tedizolid treatment. This study showed that short-course, 6-day treatment with tedizolid was as effective as 10 days of linezolid in treating ABSSSI in IDUs, which suggests that tedizolid (including exclusively oral treatment) is a suitable treatment option in this complex patient population.

\section{ACKNOWLEDGEMENTS}

Funding. This work was supported by Merck \& Co., Inc., Kenilworth, NJ, USA. This support included funding for the clinical trials as well as journal processing fees. In collaboration with the authors, employees of the study sponsor were involved in study design, data analysis, interpretation of results, and review of the manuscript. All authors had full access to all of the data in this study and take complete responsibility for the integrity of the data and accuracy of the data analysis.

Medical Writing and Editorial Assistance. Editorial and medical writing assistance in the preparation of this manuscript was provided by Sally Mitchell, PhD (Apothecom, Yardley, PA). Support for this assistance was funded by Merck \& Co., Inc., Kenilworth, NJ, USA.

Authorship. All named authors meet the International Committee of Medical Journal Editors (ICMJE) criteria for authorship for this manuscript, take responsibility for the integrity of the work as a whole, and have given final approval to the version to be published.

Author Contributions. Gregory J. Moran was involved in drafting the manuscript, data acquisition, interpretation of the results and critical review and revision of the manuscript for important intellectual content. Carisa De Anda was involved in drafting the manuscript, study conception and design, data analysis, interpretation of the results and critical review and revision of the manuscript for important intellectual content. Anita F. Das was involved in drafting the manuscript, study conception and design, data analysis, interpretation of the results and critical review and revision of the manuscript for important intellectual content. Sinikka Green was involved in drafting the manuscript, data acquisition, interpretation of the results and critical review and revision of the manuscript for important intellectual content. Purvi Mehra was involved in drafting the manuscript, data acquisition and critical review and revision of the manuscript for important intellectual content. Philippe Prokocimer was involved in drafting the manuscript, study conception and design, interpretation of the results and critical review and revision of the manuscript for important intellectual content. 
All authors provided final approval of the version to be published. All authors agree to be accountable for all aspects of the work in ensuring that questions related to the accuracy or integrity of any part of the work are appropriately investigated and resolved.

Previous Presentation. Presented at IDWeek, October 8-12, 2014, Philadelphia, PA, USA.

Disclosures. Gregory J. Moran reports receiving grant support from Trius Therapeutics (now Merck \& Co., Inc.) during the conduct of the study; he also reports receiving grants and consultancy fees from Allergan. Carisa De Anda is a current employee of Merck Sharp \& Dohme Corp., a subsidiary of Merck \& Co., Inc., Kenilworth, NJ, USA and may hold stock in the company. Philippe Prokocimer is a current employee of AntibioTx A/S, San Diego, CA, USA and a past employee of Merck \& Co., Inc., Kenilworth, NJ, USA. Anita F. Das is a current employee of ADStat, Guerneville, CA, USA and was an employee of InClin and a consultant to Cubist Pharmaceuticals (now Merck \& Co., Inc.) at the time this study was conducted and was compensated for supporting this research; she is also a consultant to Achaogen, Cempra, Cerexa, Contrafect, Durata, Melinta, Nabriva, Paratek, Tetraphase, Theravance, Wockhardt and Zavante. Sinikka Green and Purvi Mehra have nothing to disclose.

Compliance with Ethics Guidelines. All procedures followed were in accordance with the ethical standards of the responsible committee on human experimentation (institutional and national) and with the Helsinki Declaration of 1964, as revised in 2013. Informed consent was obtained from all patients for being included in the study. The ESTABLISH-1 and ESTABLISH-2 (ClinicalTrials.gov numbers NCT01170221 and NCT01421511) phase 3 studies received institutional review board approval.

Data Availability. Merck \& Co., Inc.'s data sharing policy, including restrictions, is available at http://engagezone.merck.com/ds documentation.php. Requests for access to the study data can be submitted through the EngageZone site or via email to dataaccess@merck.com.

Open Access. This article is distributed under the terms of the Creative Commons Attribution-NonCommercial 4.0 International License (http://creativecommons.org/licenses/ by-nc/4.0/), which permits any noncommercial use, distribution, and reproduction in any medium, provided you give appropriate credit to the original author(s) and the source, provide a link to the Creative Commons license, and indicate if changes were made.

\section{REFERENCES}

1. Edelsberg J, Taneja C, Zervos M, Haque N, Moore C, Reyes K, et al. Trends in US hospital admissions for skin and soft tissue infections. Emerg Infect Dis. 2009;15:1516-8.

2. Mertz D, Viktorin N, Wolbers $M$, Laifer $G$, Leimenstoll B, Fluckiger U, et al. Appropriateness of antibiotic treatment in intravenous drug users, a retrospective analysis. BMC Infect Dis. 2008;8:42.

3. Gordon RJ, Lowy FD. Bacterial infections in drug users. N Engl J Med. 2005;353:1945-54.

4. Bassetti S, Battegay M. Staphylococcus aureus infections in injection drug users: risk factors and prevention strategies. Infection. 2004;32:163-9.

5. Lloyd-Smith E, Wood E, Zhang R, Tyndall MW, Montaner JS, Kerr T. Risk factors for developing a cutaneous injection-related infection among injection drug users: a cohort study. BMC Public Health. 2008;8:405

6. Deutscher M, Perlman DC. Why some injection drug users lick their needles: a preliminary survey. Int J Drug Policy. 2008;19:342-5.

7. Binswanger IA, Kral AH, Bluthenthal RN, Rybold DJ, Edlin BR. High prevalence of abscesses and cellulitis among community-recruited injection drug users in San Francisco. Clin Infect Dis. 2000;30:579-81.

8. Murphy EL, DeVita D, Liu H, Vittinghoff E, Leung $\mathrm{P}$, Ciccarone $\mathrm{DH}$, et al. Risk factors for skin and soft-tissue abscesses among injection drug users: a case-control study. Clin Infect Dis. 2001;33: $35-40$. 
9. Huang $\mathrm{H}$, Cohen $\mathrm{SH}$, King JH, Monchaud C, Nguyen H, Flynn NM. Injecting drug use and community-associated methicillin-resistant Staphylococcus aureus infection. Diagn Microbiol Infect Dis. 2008;60:347-50.

10. Summanen PH, Talan DA, Strong C, McTeague M, Bennion R, Thompson JE Jr, et al. Bacteriology of skin and soft-tissue infections: comparison of infections in intravenous drug users and individuals with no history of intravenous drug use. Clin Infect Dis. 1995;20(suppl 2):S279-82.

11. Dausey DJ, Desai RA. Psychiatric comorbidity and the prevalence of HIV infection in a sample of patients in treatment for substance abuse. J Nerv Ment Dis. 2003;191:10-7.

12. Mackesy-Amiti ME, Donenberg GR, Ouellet LJ. Prevalence of psychiatric disorders among young injection drug users. Drug Alcohol Depend. 2012;124:70-8.

13. Hellard M, Sacks-Davis R, Gold J. Hepatitis C treatment for injection drug users: a review of the available evidence. Clin Infect Dis. 2009;49:561-73.

14. Brown SD, Traczewski MM. Comparative in vitro antimicrobial activities of torezolid (TR-700), the active moiety of a new oxazolidinone, torezolid phosphate (TR-701), determination of tentative disk diffusion interpretive criteria, and quality control ranges. Antimicrob Agents Chemother. 2010;54:2063-9.

15. Schaadt R, Sweeney D, Shinabarger D, Zurenko G. In vitro activity of TR-700, the active ingredient of the antibacterial prodrug TR-701, a novel oxazolidinone antibacterial agent. Antimicrob Agents Chemother. 2009;53:3236-9.

16. Thomson KS, Goering RV. Activity of tedizolid (TR700) against well-characterized methicillin-resistant Staphylococcus aureus strains of diverse epidemiological origins. Antimicrob Agents Chemother. 2013;57:2892-5.

17. Prokocimer P, De Anda C, Fang E, Mehra P, Das A. Tedizolid phosphate vs. linezolid for treatment of acute bacterial skin and skin structure infections: the ESTABLISH-1 randomized trial. JAMA. 2013;309:559-69.

18. Moran GJ, Fang E, Corey GR, Das AF, De Anda C, Prokocimer P. Tedizolid for 6 days versus linezolid for 10 days for acute bacterial skin and skin-structure infections (ESTABLISH-2): a randomised, double-blind, phase 3, non-inferiority trial. Lancet Infect Dis. 2014;14:696-705.

19. Shorr AF, Lodise TP, Corey GR, De Anda C, Fang E, Das AF, et al. Analysis of the phase 3 ESTABLISH trials: tedizolid versus linezolid in acute bacterial skin and skin structure infection. Antimicrob Agents Chemother. 2015;59:864-71.

20. US Department of Health and Human Services. Center for Drug Evaluation and Research (CDER). Guidance for industry: acute bacterial skin and skin structure infections: developing drugs for treatment. 2013. http://www.fda.gov/downloads/Drugs/ .../Guidances/ucm071185.pdf. Accessed April 27, 2017.

21. European Medicines Agency. Addendum to the guideline on the evaluation of medicinal products indicated for treatment of bacterial infections. 2013. http://www.ema.europa.eu/docs/en_GB/ document_library/Scientific_guideline/2013/11/ WC500153953.pdf. Accessed April 27, 2017.

22. Miettinen $\mathrm{O}$, Nurminen M. Comparative analysis of two rates. Stat Med. 1985;4:213-26.

23. Tirelli U, Vaccher E, Carbone A, Volpe R, De Paoli P, Santini G, et al. Persistent generalized lymphadenopathy: clinical characteristics of a lymphadenopathy syndrome in intravenous drug abusers. AIDS Res. 1986;2:227-30.

24. Lloyd-Smith E, Kerr T, Hogg RS, Li K, Montaner JS, Wood E. Prevalence and correlates of abscesses among a cohort of injection drug users. Harm Reduct J. 2005;2:24.

25. Azar P, Wood E, Nguyen P, Luma M, Montaner J, Kerr T, et al. Drug use patterns associated with risk of non-adherence to antiretroviral therapy among HIV-positive illicit drug users in a Canadian setting: a longitudinal analysis. BMC Infect Dis. 2015;15:193.

26. Colosia A, Pladevall-Vilia M, Khan S, Kauf T. Simple and short-duration dosing schedules improve patient adherence to oral anti-infectives. In: Presented at: 49th midyear clinical meeting of the American Society of Health-system Pharmacists, Anaheim, December 7-11, 2014.

27. Boyer EW, Shannon M. The serotonin syndrome. N Engl J Med. 2005;352:1112-20.

28. Durkin MJ, Corey GR. New developments in the management of severe skin and deep skin structure infections-focus on tedizolid. Ther Clin Risk Manag. 2015;11:862.

29. Flanagan S, Bartizal K, Minassian SL, Fang E, Prokocimer P. In vitro, in vivo, and clinical studies of tedizolid to assess the potential for peripheral or central monoamine oxidase interactions. Antimicrob Agents Chemother. 2013;57:3060-6. 